\title{
Schlafen 12 Reduces the Aggressiveness of Triple Negative Breast Cancer through Post-Transcriptional Regulation of ZEB1 That Drives Stem Cell Differentiation
}

\author{
Sarmad Al-Marsoummia Emilie Vomhof-DeKrey ${ }^{a, b} \quad$ Marc D. Basson $n^{a, b, c}$ \\ aDepartment of Biomedical Sciences, University of North Dakota School of Medicine and the Health \\ Sciences, Grand Forks, ND, USA, 'DDepartment of Surgery, University of North Dakota School of \\ Medicine and the Health Sciences, Grand Forks, ND, USA, 'Department of Pathology, University of \\ North Dakota School of Medicine and the Health Sciences, Grand Forks, ND, USA
}

\section{Key Words}

Schlafen12 $\cdot$ TNBC $・$ Breast cancer stem cell $•$ Differentiation

\begin{abstract}
Background/Aims: Schlafen12 (SLFN12) promotes human intestinal and prostatic epithelial differentiation. We sought to determine whether SLFN12 reduces triple-negative breast cancer (TNBC) aggressiveness. Methods: We validated bioinformatics analyses of publicly available databases by staining human TNBC. After virally overexpressing or siRNAreducing SLFN12 in TNBC cell lines, we measured proliferation by CCK- 8 assay, invasion into basement-membrane-coated pores, mRNA by q-RT-PCR and protein by Western blotting. Flow cytometry assessed proliferation and stem cell marker expression, and sorted CD44+/ CD24- cells. Stemness was also assessed by mammosphere formation, and translation by click-it-AHA chemistry. Results: SLFN12 expression was lower in TNBC tumors and correlated with survival. SLFN12 overexpression reduced TNBC MDA-MB-231, BT549, and Hs578T proliferation. In MDA-MB-231 cells, AdSLFN12 reduced invasion, promoted cell cycle arrest, increased $\mathrm{E}$-cadherin promoter activity, $\mathrm{mRNA}$, and protein, and reduced vimentin expression and protein. SLFN12 knockdown increased vimentin. AdSLFN12 reduced the proportion of MDA-MB-231 CD44 ${ }^{+}$CD24 cells, with parallel differentiation changes. SLFN12 overexpression reduced MDA-MB-231 mammosphere formation. SLFN12 overexpression decreased ZEB1 and Slug protein despite increased ZEB1 and Slug mRNA in all three lines. SLFN12 overexpression accelerated MDA-MB-231 ZEB1 proteasomal degradation and slowed ZEB1 translation. SLFN12 knockdown increased ZEB1 protein. Coexpressing ZEB1 attenuated the SLFN12 effect on E-cadherin mRNA and proliferation in all three lines. Conclusion: SLFN12 may reduce TNBC aggressiveness and improve survival in part by a post-transcriptional decrease in ZEB1 that promotes TNBC cancer stem cell differentiation.




\section{Cellular Physiology Cell Physiol Biochem 2019;53:999-1014 \\ \begin{tabular}{ll|l} 
and Biochemistry & $\begin{array}{l}\text { DOl: } 10.33594 / 000000191 \\
\text { Published online: } 16 \text { December } 2019\end{array}$ & $\begin{array}{l}\text { C } 2019 \text { The Author(s). Published by } \\
\text { Cell Physiol Biochem Press GmbH\&Co. KG }\end{array}$
\end{tabular}}

Al-Marsoummi et al.: Schlafen12 Regulates Breast Cancer Stemness

\section{Introduction}

Triple-negative breast cancer (TNBC) is an aggressive basal-like subtype of breast cancer that does not express estrogen or progesterone receptors, or human epidermal growth factor receptor 2 (HER2) [1]. TNBC constitutes $20 \%$ of breast cancer [2]. Because TNBC lacks hormonal receptors; conventional cytotoxics, and surgery remain the standard of care [2, 3]. However, TNBC is aggressive, chemoresistant, and radioresistant [1, 2]. Understanding the signals that drive TNBC aggressiveness may facilitate targeted therapy. TNBC is poorly differentiated and enriched in CD44 ${ }^{+} \mathrm{CD} 24-$ cells, which are breast cancer stem cells (BCSCs) that drive its aggressive treatment-resistant phenotype [4, 5]. Diverse transcription factors influence epithelial to mesenchymal transition (EMT) [6], maintain the poorly differentiated state of TNBC, and maintain the BCSCs population. In particular, ZEB1 is a master EMT regulator that correlates with treatment-resistance [6-9], but how ZEB1 is regulated is incompletely understood.

Schlafen proteins are grouped based upon structure and size into short, intermediate, and long [10]. Schlafen12 (SLFN12) is the only human intermediate Schlafen; slfns 3 and 4 are rodent intermediate Schlafens [11]. Long Schlafens (SLFN5, SLFN11, and SLFN13) reduce proliferation and invasiveness in human cancers $[12,13]$ by modulating transcription and contain a nuclear targeting sequence. Intermediate SLFN12 and murine Slfn3 [14] lack this sequence and act in the cytosol [15]. In fact, Slfn3 retains activity even when excluded from the nucleus [15]. SLFN12 induces intestinal epithelial differentiation [16], reduces prostate cancer proliferation, and induces prostate cancer differentiation [13]. Slfn3 is linked to cell growth, maturation, and differentiation, and slows proliferation [17-19] SLFN12 has never previously been studied in breast cancer, although it sensitizes cancer cells to specific phosphodiesterase 3A inhibitors [20] and is expressed in human breast tissue [21].

Our analysis of a publicly available [22] dataset from a cohort of patients with breast cancer demonstrated that higher SLFN12 expression correlated with longer survival in TNBC patients. We therefore further investigated how this might occur using TNBC cell lines. We demonstrated that adenoviral SLFN12 overexpression reduced proliferation and invasion, while inducing TNBC differentiation. SLFN12 knockdown exhibited the reverse effect. Moreover, SLFN12 reduced the proportion of CD44 ${ }^{+} \mathrm{CD} 24$ cancer stem cells within the cell population and reduced mammosphere formation consistent with such biologic effects. We then further demonstrated that SLFN12 modulates E-cadherin expression and proliferation by regulating both translation and proteasomal degradation of ZEB1.

\section{Materials and Methods:}

\section{Cells and reagents}

Cell lines were from the American Tissue Culture Collection (ATCC). MDA-MB-231 cells were cultured in DMEM with 10\% fetal bovine serum (FBS) and 5\% Penicillin/Streptomycin. BT549 and Hs578T cells were cultured per ATCC recommendations. Cycloheximide, bortezomib, carfilzomib, and MG132 were from Selleckchem (Houston, TX). Primers are listed in Tables 1 and 2, and antibodies are listed in Table 3.

\section{Viral constructs}

AdSLFN12 was from Applied Biological Materials (Richmond, BC, Canada) utilizing a pAdeno vector, CMV promoter, and human Slfn12 insert (accession \# NM_018042). AdZEB1 was from Vector Biolabs (\#ADV-228176, Malvern, PA). V5-tagged SLFN12 and control lentiviruses were constructed in HEK293T cells as described [16].

siRNA and virus studies

70-80\% confluent MDA-MB-231, BT549, Hs578T cells in six-well plates were transduced at 10004000 particles/cell with AdCMV control, AdSLFN12 or AdZEB1 for twenty-four hours in 1ml complete DMEM (for MDA-MB-231, Hs578T cells) or RPMI1640 (for BT549 cells). Twenty-four hours after infection, 
Table 1. Primer-Prob qPCR primers: All Primers were obtained from Integrated DNA Technology (IDT, Coralville, IA, USA)

\begin{tabular}{|c|c|c|c|}
\hline Gene & Forward & Reverse & Probe \\
\hline Human SLFN12 & 5'-GGGAGCAGGTAATGACGTATTTATT-3' & 5'- CAGTTGACCAGGAAGGAATGG-3' & 5'-/56-FAM/ATCCAGTTC/ZEN/ATGGTGGAGGCTGAA/3IABkFQ/-3' \\
\hline Human Vimentin & 5'- GTGAATCCAGATTAGTTTCCCTCA-3' & 5'- CAAGACCTGCTCAATGTTAAGATG-3' & 5'-/5Cy5/CGCCTTCCAGCAGCTTCCTGTA/3IAbRQSp/-3' \\
\hline Human ZEB1 & 5'- TACTGTACATCCTGCTTCATCTG-3' & 5'-GAGGAGCAGTGAAAGAGAAGG-3' & 5'- /56-FAM/TCCTTTCCT/ZEN/GTGTCATCCTCCCAG/3IABkFQ/-3' \\
\hline Human CDH1 & 5'- GTCTGTCATGGAAGGTGCTC-3' & 5'- CTGAGGATGGTGTAAGCGATG-3' & 5'-/56-FAM/AGACGCGGA/ZEN/CGATGATGTGAACAC/3IABkFQ/-3' \\
\hline Human HPRT & 5'-GCGATGTCAATAGGACTCCAG-3' & 5'- TTGTTGTAGGATATGCCCTTGA-3' & 5'- /5HEX/AGCCTAAGA/ZEN/TGAGAGTTCAAGTTGAGTTTGG/3IABkFQ/-3' \\
\hline Human CD24 & 5'- CAATGTCAAATCCAAAGCCTCA -3' & 5'- CTCAACGTATTGTTTCGACAGC -3' & 5'-/56-FAM/TCAGTTCCC/ZEN/AAGAGAACAGCAATAGCTC/3IABkFQ/ -3' \\
\hline Human CD44 & 5'- GAGATGCTGTAGCGACCATT -3' & 5'- GACACCATGGACAAGTTTTGG -3' & 5'-/5Cy5/TGGAATACACCTGCAAAGCGGC/3IAbRQSp/-3' \\
\hline
\end{tabular}

the medium was changed, and cells were harvested at 72 or 96 hours.

For lentivirus studies, 500ul viral HEK-239T supernatant was added to $70-80 \%$ confluent cells with $1.5 \mathrm{ml}$ OptiMEM medium containing $1.5 \mathrm{ul}$ polybrene transfection reagent (Millipore-Sigma, Burlington, MA, \#TR-1003-G) at 10ug/ml. Two days later, media was replaced with fresh DMEM media with 10\% fetal bovine serum and $10 \mu \mathrm{M}$ Blasticidin-s HCL (Gibco, Waltham, MA, \#A11139-03) and cells were cultured for three weeks for selection.

For siRNA studies, 300, 000 cells/well were plated into six-well plates before transfection with non-targeting siNT5 (100nM) (Dharmacon, D-001210-05-20) or SMARTpool siSLFN12 (100nM) (Dharmacon, L-018142-02-0020) using Lipofectamine RNAiMAX (Thermo Fisher, Waltham, MA, \#13778150) transfection reagent (7.5ul/well) formulated in Opti-MEM medium. The mixture was added gradually into $1 \mathrm{ml}$ of complete medium/well. Medium was changed after eight hours and experiments terminated after 72 hours.

\section{Cell Viability}

Cell viability was assayed using the cell-counting-kit-8 (CCK-8), (Dojindo Molecular Technology, Rockville, MD). Cells were seeded in 96-well plates at 3000 cells/well (for MDA-MB-231 cells) or at 5000 cells/well for BT549, and Hs578T cells. After 24 hours, cells were infected with adenoviral vectors at 4000 particles/cell (MDA-MB-231 and Hs578T) or 1000 particles/cell (BT549). Absorbance was measured at $450 \mathrm{~nm}$ with a Biotek Epoch spectrophotometer (Winooski, VT).

\section{Cell Invasion}

MDA-MB-231 cells were seeded in 6-well plates at 200, 000 cells/well overnight, infected with 4000 particles/cell AdSLFN12 or control AdCMV and trypsinized and counted after 64 hours. 300, 000 cells were seeded into invasion inserts from Cell Biolab (\# CBA-110). Inserts were incubated for 8 hours and processed per manufacturer's protocol. The optical density of extracted inserts was read at 560nM using a Biotek Epoch spectrophotometer.

\section{Mammosphere assay}

10, 000 cells/well were seeded into ultra-low adherent six-well plates (Corning, \#27145) with $2 \mathrm{ml}$ of MammoCult Human Medium Kit (STEMCELL Technologies, Cambridge, MA, \#05620) and photographed after seven days with an Evos-FL cell imaging microscope (Thermo Fisher) at 40x. The four corners and center of each well were imaged and processed through Image (NIH) to calculate mammosphere size. Mammospheres $<40 \mu \mathrm{m}$ in diameter were excluded per published protocols [23]. 


\section{Cellular Physiology Cell Physiol Biochem 2019;53:999-1014 \begin{tabular}{ll|l} 
and Biochemistry & $\begin{array}{l}\text { DOl: 10.33594/000000191 } \\
\text { Published online: 16 December 2019 }\end{array}$ & $\begin{array}{l}\text { O 2019 The Author(s). Published by } \\
\text { Cell Physiol Biochem Press GmbH\&Co. KG }\end{array}$ \\
\cline { 2 - 3 }
\end{tabular} \\ Al-Marsoummi et al.: Schlafen12 Regulates Breast Cancer Stemness}

Flow Cytometry

Flow cytometry was acquired on a BD Symphony flow cytometer or a FACSAria cell sorter (BD, San Jose, CA) and analyzed with FlowJo software (TreeStar, Ashland OR).

\section{Cell Proliferation}

6-10x10 ${ }^{6}$ cells were incubated with $1 \mathrm{ml}$ PBS with $5 \mu \mathrm{M}$ Tag-IT violet dye (BioLegend, \#425101) for 30 minutes at room temperature. The reaction was terminated by adding $5 \mathrm{ml}$ of DMEM with $10 \%$ FBS. Cells were centrifuged, resuspended in fresh DMEM, and seeded into six-well plates at 200, 000 cells/well for one day before infection with AdSLFN12 or control AdCMV. Twenty-four hours later, the medium was changed. Seventy-two hours after infection, cells were trypsinized and resuspended in $600 \mu$ FACS buffer (PBS+5\%FBS).

\section{Cell cycle}

Cells were serum-starved overnight and infected with AdSLFN12 or AdCMV. After 72 hours, cells were trypsinized and incubated for 30 minutes in the dark with $5 \mu \mathrm{M}$ Vybrant dye cycler (Thermo Fisher, \#V35003).

\section{CD44/CD24 labeling}

Cells were seeded into 6-well plates at 200, 000 cells/well. After 24 hours, cells were infected with AdSLFN12 or AdCMV (4000 particles/cell). Ninety-six hours after infection, cells were trypsinized, PBSwashed, pretreated with Trustain FCX FC-blocker (Biolegend, \#422302) at 1:25 dilution for 5 minutes on ice, washed with FACS buffer (PBS+5\%FBS), and labeled with Human FITC-conjugated anti-CD44 and Human PE conjugated CD24 or Human APC-conjugated CD24 at $4^{\circ} \mathrm{C}$ for 30 minutes in the dark followed by washing with FACS buffer.

\section{Cell sorting}

Cells were blocked with FC-blocker (Trustain FCX, Biolegend) at 1:25 dilution for 5 minutes on ice and labeled with FITC conjugated-human CD44 and APC conjugated-human CD24 for 30 minutes at $4 \mathrm{C}^{\circ}$. Purity sort of $\mathrm{CD}_{4} 4^{+} \mathrm{CD} 24$ - cells was performed using the BD FACSAria IIu into DMEM with $20 \%$ FBS. Cells were seeded into 6-well plates at 200, 000 cells/well. After 48 hours, AdSLFN12 or AdCMV was added (4000 particles/cell). 96 hours later, cells were processed for CD44 and CD24 labeling as above and cell cycle analysis with FxCycle PI/RNase (Thermo Fisher, \#F10797).

\section{Immunoblotting}

Western blotting was as previously [16] except that 8\% SDS-PAGE and 1.25 hour transfer were used for Slug and Snail. Primary antibodies were: rabbit antibodies to Schlafen 12 (monoclonal), ZEB1, vimentin, Slug, and beta-actin, and mouse antibodies to E-cadherin, Snail, GAPDH monoclonal. Membranes were incubated with species-specific IRDye-secondary antibodies 800CW and 680RD (LI-COR), imaged using a LI-COR Odyssey CLx, and analyzed using image studio software (LI-COR).

\section{$q P C R$}

RNA was isolated and PCR performed as described previously [16]. Expression was calculated from threshold cycle (Ct) values by $2^{-\Delta \Delta C t}$ using RPLP0/HPRT as reference genes.

\section{Promoter Luciferase Assay}

One day after seeding 300, 000 cells/well, cells were transduced using Lipofectamine3000 (Thermo Fisher, \#L3000015) per manufacturer's protocols with 2000ng/well of E-cadherin promotor proE-cad670Luc vector (Addgene, \#42083) and 400ng/well of pNL1.1.TK[Nluc/TK] vector (Promega, \#N1501).

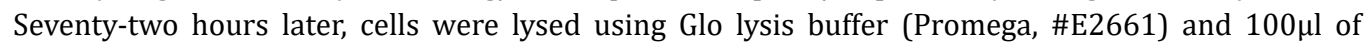
lysate was loaded into 96 well plates in triplicate. Luciferase activity was measured normalized to Nluc/ TK luciferase activity using Nano-Glo Dual-Luciferase Reporter Assay System (Promega, \#N1610) per manufacturer's protocol, using a Biotek microplate reader FLX800 (Winooski, VT). 
ZEB1 translation

One day after seeding 300, 000 cells/well, cells were infected with AdSLFN12 or AdCMV. Sixty hours later, the medium was replaced with methionine-free medium (Thermo Fisher, \#21013024) for two hours.

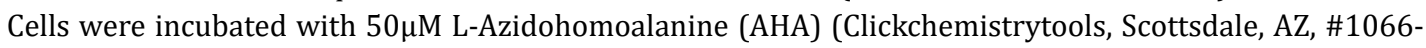
25) for 8 hours and lysed in NP-40 lysis buffer (Thermo Fisher, \#FNN0021) with 1\%SDS. AHA was detected using click chemistry with 50 $\mu \mathrm{M}$ Biotin Alkyne (Clickchemistrytools, \#1266-5) with Click-\&-Go ${ }^{\mathrm{TM}}$ Protein Reaction Buffer Kit (Clickchemistrytools, \#1262) per manufacturer's protocol. After click chemistry, ZEB1 was immunoprecipitated using rabbit anti-ZEB1 and protein-A magnetic beads (Bio-Rad Laboratories, \#161-4013). Proteins were resolved by $10 \%$ SDS-PAGE, transferred to $0.45 \mu \mathrm{m}$ nitrocellulose membrane, and blocked with Odyssey blocking buffer (LI-COR, Lincoln, NE, \#927-50010). Biotin was labeled with IRDye 800CW Streptavidin per manufacturer's protocol (LI-COR, \#926-32230) and ZEB1 was labeled with IRDye 680LT Donkey anti-Rabbit IgG Secondary Antibody. Images were acquired using a LI-COR-Clx and analyzed using Image Studio (LI-COR).

\section{Immunohistochemistry}

$10 \mu \mathrm{m}$ paraffin sections of de-identified human TNBC tissues from the IRB-approved archival repository of the Department of Pathology of the University of North Dakota, were labeled with polyclonal rabbit antischlafen12 at 1:200 and stained with a Bondmax stainer (Leica, Germany) using program Mod F, AR1 20, and a BOND Polymer Refine detection kit (Leica, \#DS9800). Images were acquired with a Zeiss Axioskop microscope (Germany) using 200x magnification.

\section{Statistical Analysis}

Data are expressed as mean \pm SEM and analyzed using GraphPad prism v8 via Student $t$ test with Bonferroni correction when needed unless stated otherwise. All experiments were repeated at least three times within the assay linear range.

\section{Results}

\section{Schlafen12 correlates with better survival in TNBC}

In publicly available datasets [24] of gene expression profiles of TNBC patients, higher SLFN12 expression correlated with recurrence-free survival (RFS) (Hazard ration=0.49, $\mathrm{n}=255, \mathrm{p}<0.001$ ) (Fig. 1A). In contrast, expression of other human Schlafens (SLFN5, 11, and 13) did not correlate with survival in TNBC from the same dataset (not shown). Interestingly, SLFN12 did not correlate with survival in patients with breast cancers expressing estrogen and progesterone receptors in the same dataset (Hazard ration=0.78, $n=559, p=0.19$ ) (Fig. 1B). Analyzing a second dataset from TCGA demonstrated that higher SLFN12 mRNA expression correlated with favorable Scarff Bloom and Richardson grade (Fig. 1C) and favorable Nottingham Prognostic Index (Fig. 1D) [25]. Indeed, a third dataset of RNAseq data of breast cancer tissue samples [26] demonstrated reduced mRNA expression of SLFN12 in breast adenocarcinoma and invasive breast lobular carcinoma vs. both normal and adjacent stroma (Fig. 1E) while we observed reduced SLFN12 protein immunoreactivity in malignant vs. benign adjacent mammary tissue in our own samples (Fig. 1F).

\section{Schlafen12 overexpression reduces MDA-MB-231 proliferation and invasion}

To explore the potential mechanism of the effect of high SLFN12 expression on TNBC, we studied the well characterized and highly aggressive and invasive MDA-MB-231, in addition to BT549, and Hs578T as TNBC models. MCF-7 cells modeled estrogen/progesterone receptor positive breast cancer. Exogenous SLFN12 expression (Supplementary Fig. 1 - for all supplemental material see www.cellphysiolbiochem.com) using an adenoviral vector (AdSLFN12) slowed MDA-MB-231 proliferation vs. cells infected with empty vector (AdCMV) as a control $(6.14 \pm 0.73$ fold vs. $9.05 \pm 0.81$ fold) (Fig. 2A). The anti-proliferative effect of SLFN12 was replicated in two in BT549 and Hs578T cells (Fig. 2A). In contrast, SLFN12 overexpression did not inhibit $\mathrm{ER}^{+} \mathrm{PR}^{+} \mathrm{MCF}-7$ proliferation (Fig. 2B). 


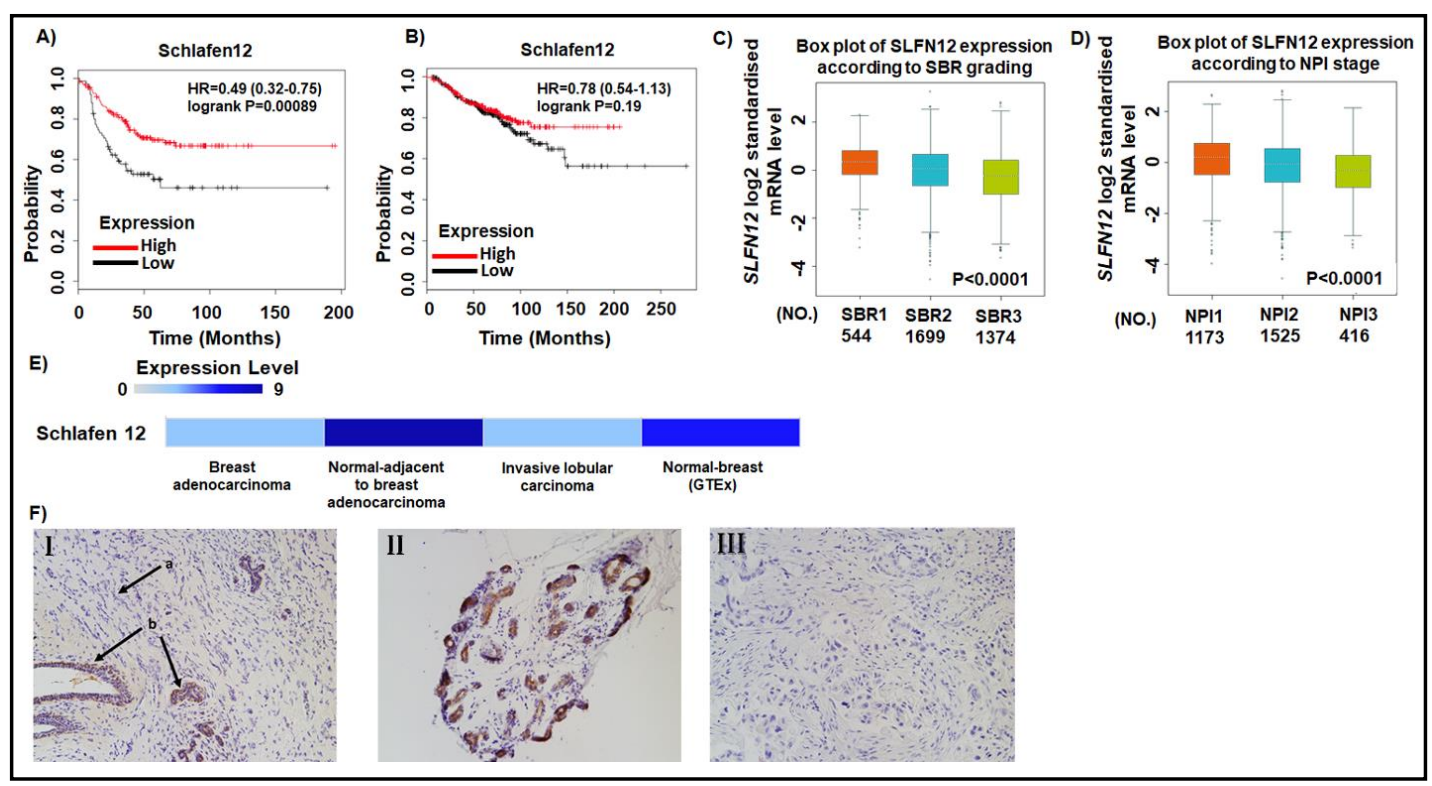

Fig. 1. Schlafen12 expression correlates with survival in triple negative breast cancer. A) Survival analysis of publicly available data set of cohorts of patients with triple negative breast cancer shows that SLFN12 mRNA expression correlates with relapse free survival (http://kmplot.com/analysis/; $n=255, p<0.01$ ). B) Survival analysis shows that Schlafen 12 mRNA expression does not correlate with relapse free survival in patients with breast cancers expressing estrogen and progesterone receptors (http://kmplot.com/ analysis/; $n=559, \mathrm{p}=0.19$ ). C) Higher SLFN12 mRNA expression correlated with favorable Scarff Bloom and Richardson grade (http://bcgenex.centregauducheau.fr/, $n=3627, \mathrm{p}<0.0001$ ). D) Higher SLFN12 mRNA expression correlated with favorable Nottingham Prognostic Index in breast cancer (http://bcgenex. centregauducheau.fr/, n=3114, p<0.0001). E) RNAseq analysis of publicly available data (https://www.ebi. ac.uk/gxa/home) of patients with breast cancers demonstrates that SLFN12 mRNA expression is reduced in breast cancer tissue vs. normal breast tissue or adjacent benign stroma. F) Representative images of Schlafen12 immunohistolabeling (brown stain) demonstrate reduced SLFN12 labeling intensity in triple negative breast cancer tissue (Ia, III) vs. benign tissue (Ib, II), (n=13, 200x).

To confirm that the reduction in MDA-MB-231 number reflected slower proliferation, we analyzed MDA-MB-231 proliferation by flow cytometry using a Tag-IT Violet intracellular protein binding dye assay. AdSLFN12 reduced the fraction of proliferating MDA-MB-231

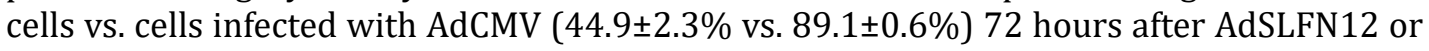
AdCMV infection (Fig. 2C).

This reduction of proliferation in MDA-MB-231 cells by SLFN12 was associated with a substantial increase in the fraction of SLFN12 overexpressing cells in G0/G1 phase vs. control cells infected with AdCMV alone (42.3 $\pm 6.1 \%$ vs. $17.1 \pm 6.7 \%$, Fig. 2D).

Exogenous SLFN12 also significantly reduced the fraction of invasive MDA-MB-231 cells

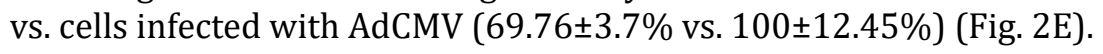

\section{Schlafen12 induces differentiation of MDA-MB-231 cells}

We next asked whether Schlafen12 can induce MDA-MB-231 differentiation. AdSLFN12 substantially increased the mRNA and protein of the epithelial marker E-cadherin (Fig. 3A, B), as well as E-cadherin promotor activity (Fig. 3C). Moreover, AdSLFN12 reduced the mesenchymal marker vimentin at both mRNA and protein levels (Fig. 3A, B). AdSLFN12 also increased mRNA levels of the epithelial luminal marker cytokeratin-18 (CK18) (Fig. 3D). Knockdown of SLFN12 using siRNA (Supplementary Fig. 2) increased vimentin mRNA and protein (Fig. 3E, 3F). Stable SLFN12-overexpressing MDA-MB-231 subclones showed increased E-cadherin and decreased vimentin mRNA (Fig. 3G). 


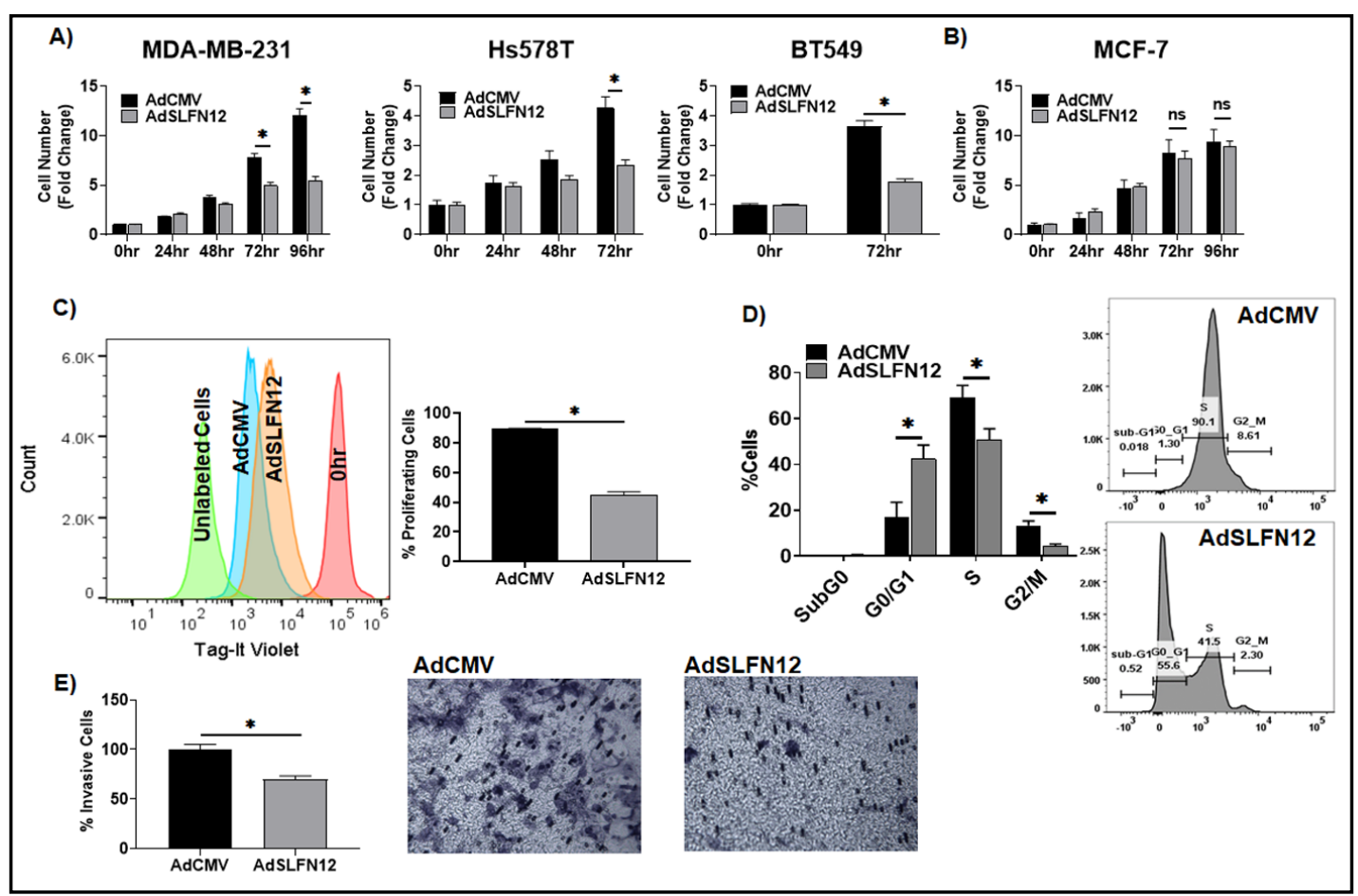

Fig. 2. Schlafen12 reduces aggressiveness in triple negative breast cancer cells. A) Exogenous Schlafen12 expression using adenoviral vector (AdSLFN12) significantly reduced proliferation at 72 hours post AdSLFN12 treatment in MDA-MB-231 $\left(n=13,{ }^{*} \mathrm{p}<0.05\right)$, BT549 $\left(\mathrm{n}=14,{ }^{*} \mathrm{p}<0.05\right)$, and Hs578T cells $(\mathrm{n}=3$, ${ }^{*} \mathrm{p}<0.05$ ) vs. empty vector (AdCMV) treatment. Data represent mean fold change in cell number normalized to zero-hour time point. B) AdSLFN12 treatment did not reduce the proliferation of MCF-7 cells vs. AdCMV treatment, data represent fold change in cell number normalized to zero-hour time point (n=6, ns=not significant). C) Representative histogram shows AdSLFN12 reduced the proliferation of MDA-MB-231 cells; analyzed by flow cytometry using Tag-IT Violet proliferation and cell tracking dye, the higher fluorescence intensity in the histogram indicates less proliferation $\left(n=3,{ }^{*} \mathrm{p}<0.01\right)$. D) Cell cycle analysis by flow cytometry using Vybrant DyeCycle of MDA-MB-231 cells in the presence of AdSLFN12 or AdCMV. Cells were synchronized at G0/G1 phase by serum starvation, and results represent data 72 hours after treatment $(n=3, * p<0.05)$. E) Colorimetric invasion assay shows that AdSLFN12 treatment inhibited the invasion of MDA-MB-231 cells after 72 hours. Data represent the percentage of change in optical density normalized to AdCMV treatment $\left(n=6,{ }^{*} p<0.05\right)$. All error bars shown represent standard error of mean.

Schlafen 12 reduces breast cancer stem cell phenotype of MDA-MB-231 cells

Since SLFN12 induced MDA-MB-231 differentiation by reducing vimentin and increasing E-cadherin, since vimentin contributes to breast cancer stem cell biology in MDA-MB-231 cells and attenuating vimentin reduces stem cell features [27], and since SLFN12 reduced MDA-MB-231 proliferation and invasion which are both features of breast cancer stem cells, we hypothesized that AdSLFN12 would change the breast cancer stem cell proportion in an MDA-MB-231 population. We classified $\mathrm{CD} 44^{+} \mathrm{CD} 24$ cells as breast cancer stem cells (BCSCs) and the remaining phenotypes (CD44+CD24+, CD44-CD24- CD44-CD24) as nonBCSCs or differentiated cells [28]. AdSLFN12 reduced the proportion of CD44 ${ }^{+} \mathrm{CD} 24{ }^{-}$cells vs. control cells infected with the empty vector AdCMV (38.66 $\pm 2.5 \%$ vs. $56.73 \pm 2.11 \%$ ) (Fig. 4A). Conversely, AdSLFN12 increased the proportion of the luminal more differentiated CD44CD24 ${ }^{+}$phenotype $(18.66 \pm 1.35 \%$ vs. $1.09 \pm 0.13 \%$ ) (Fig. $4 \mathrm{~A})$. In contrast, AdSLFN12 did not change CD44 or CD24 in ER ${ }^{+} \mathrm{PR}^{+}$MCF-7 cells (Supplementary Fig. 3). Parallel studies showed that AdSLFN12 reduced CD44 mRNA and increased CD24 mRNA (Fig. 4B). AdSLFN12 also reduced mRNA levels of CD49F and SSEA4 (Fig. 4C), which are also correlated with chemoresistance and stemness in TNBC $[29,30]$. 


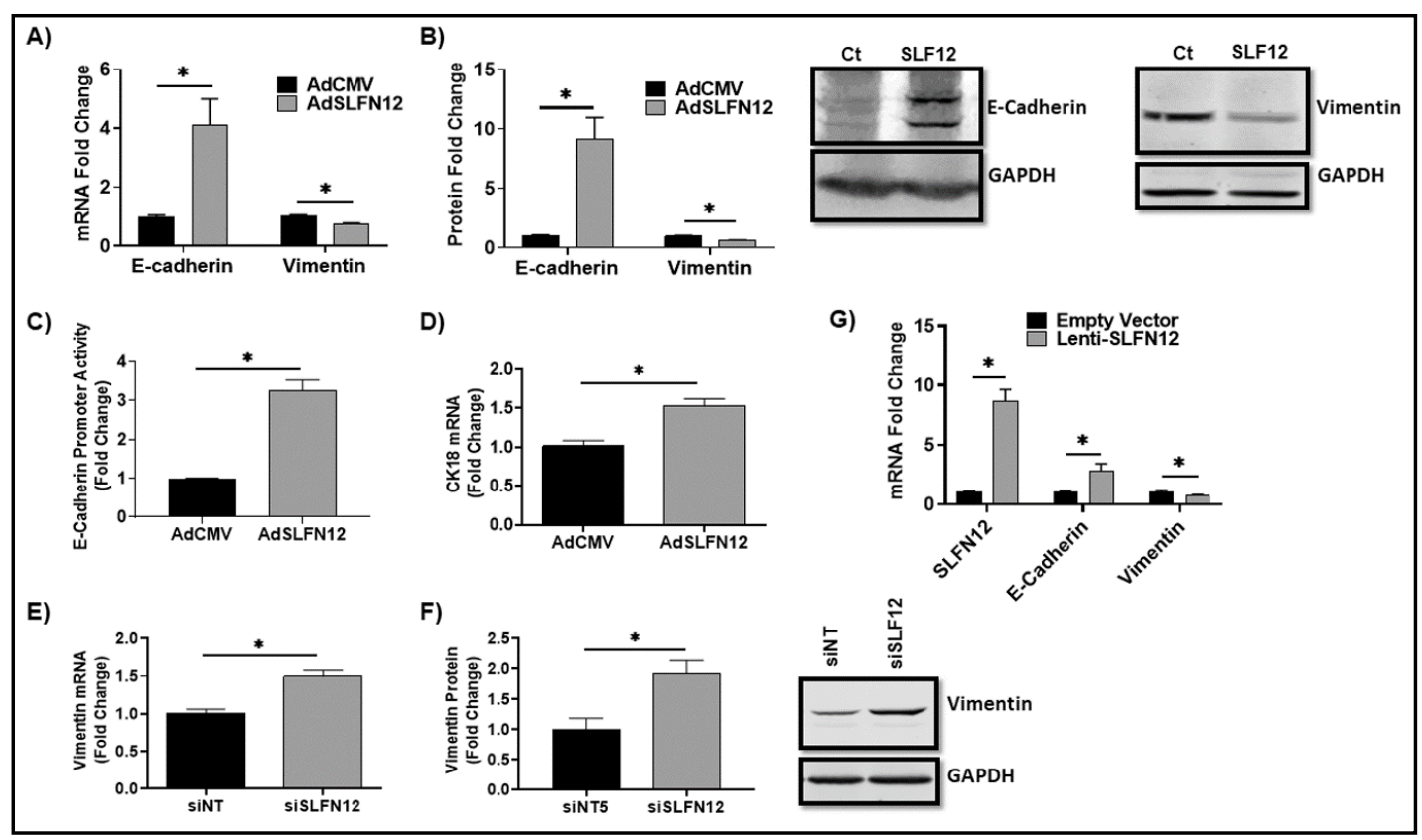

Fig. 3. Schlafen 12 induces TNBC cell differentiation. A) mRNA analysis by SYBR Green qPCR of E-cadherin $(n=27)$ and Vimentin $(n=12)$ and $B)$ protein levels of E-cadherin $(n=17)$ and Vimentin $(n=6)$ analyzed by western blot with representative blot images in MDA-MB-231 cells 72 hours after AdSLFN12 or AdCMV treatment (RPLP0, qPCR reference gene, GAPDH served as a reference protein; data normalized to AdCMV group, $\left.{ }^{*} \mathrm{p}<0.05\right)$. C) Luciferase activity of E-cadherin promoter in MDA-MB-231 cells 72 hours after treatment with AdSLFN12 or AdCMV normalized to Nluc/TK Vector $\left(n=6,{ }^{*} \mathrm{p}<0.05\right)$. D) SYBR Green qPCR analysis of CK-18 or mRNA levels in MDA-MB-231 cells 72 hours after AdSLFN12 or AdCMV treatment (RPLP0 used as a reference gene), data normalized to AdCMV group ( $\left.n=9,{ }^{*} p<0.05\right)$. E) Primer-probe qPCR analysis of vimentin mRNA levels $(n=9)$ and $F$ ) Western blot analysis with representative blot image of vimentin protein levels $(n=6)$ in MDA-MB-231 cells 72 hours after treatment with either siSLFN12 or non-targeting sequence (siNT5) as a control (HPRT served as a reference gene, GAPDH used as a reference protein, data normalized to siNT group, * $\left.{ }^{*}<0.05\right)$. G) MDA-MB-231 clones with stable SLFN12 expression generated using lentiviral vector transfection with blasticidin selection showing E-cadherin $(\mathrm{n}=11)$ and vimentin $(\mathrm{n}=7)$ mRNA levels analyzed by Primer-probe RT-qPCR. (HPRT used as a reference gene); data normalized to AdCMV group $\left({ }^{*} \mathrm{p}<0.05\right)$. All error bars shown represent standard error of mean.

Moreover, SLFN12-overexpressing MDA-MB-231 subclones displayed reduced mammosphere formation efficiency $(0.03 \pm 0.003 \%$ vs. $0.07 \pm 0.008 \%$ ) (Fig. 4D) and developed smaller mammospheres (77.69 $\pm 4.51 \mu \mathrm{m}$ vs.151.90 $\pm 7.51 \mu \mathrm{m})$ (Fig. 4E, F).

Since MDA-MB-231 cells are a heterogeneous population of differentiated and BCSCs, we asked whether SLFN12 affects BSCS directly. We FACs-sorted CD44+CD24- BCSCs and overexpressed SLFN12 using AdSLFN12. AdSLFN12 altered the phenotypic expression of CD44 and CD24, reducing the CD44+CD24- BCSC population $(37.81 \pm 9.58 \%$ to $92.57 \pm 1.78 \%$ ) and increasing the differentiated breast cancer stem cell population $(52.90 \pm 6.95 \%$ to $2.84 \pm 0.31 \%$ ) vs. sorted $\mathrm{CD} 44^{+} \mathrm{CD} 24$ cells treated with AdCMV only (Fig. 4G). AdSLFN12 changed the cell cycle distribution of the sorted CD44+CD24 cells vs. AdCMV control with most cells shifted to subG0 phase $(44.04 \pm 2.78 \%$ vs. $3.58 \pm 0.67 \%)$ (Fig. $4 \mathrm{H})$.

\section{Schlafen12 reduces $Z E B 1$ Protein}

Since epithelial genes including E-cadherin are silenced in MDA-MB-231 through EMT transcription factors [31, 32], we asked whether SLFN12 affects such factors. AdSLFN12 increased mRNA for Snail by $260 \%$, Slug by $64 \%$, and ZEB1 by $81 \%$. ZEB2 mRNA only increased by $18 \%$, which was not significant (Fig. $5 \mathrm{~A}$ ). In contrast to the mRNA results, 


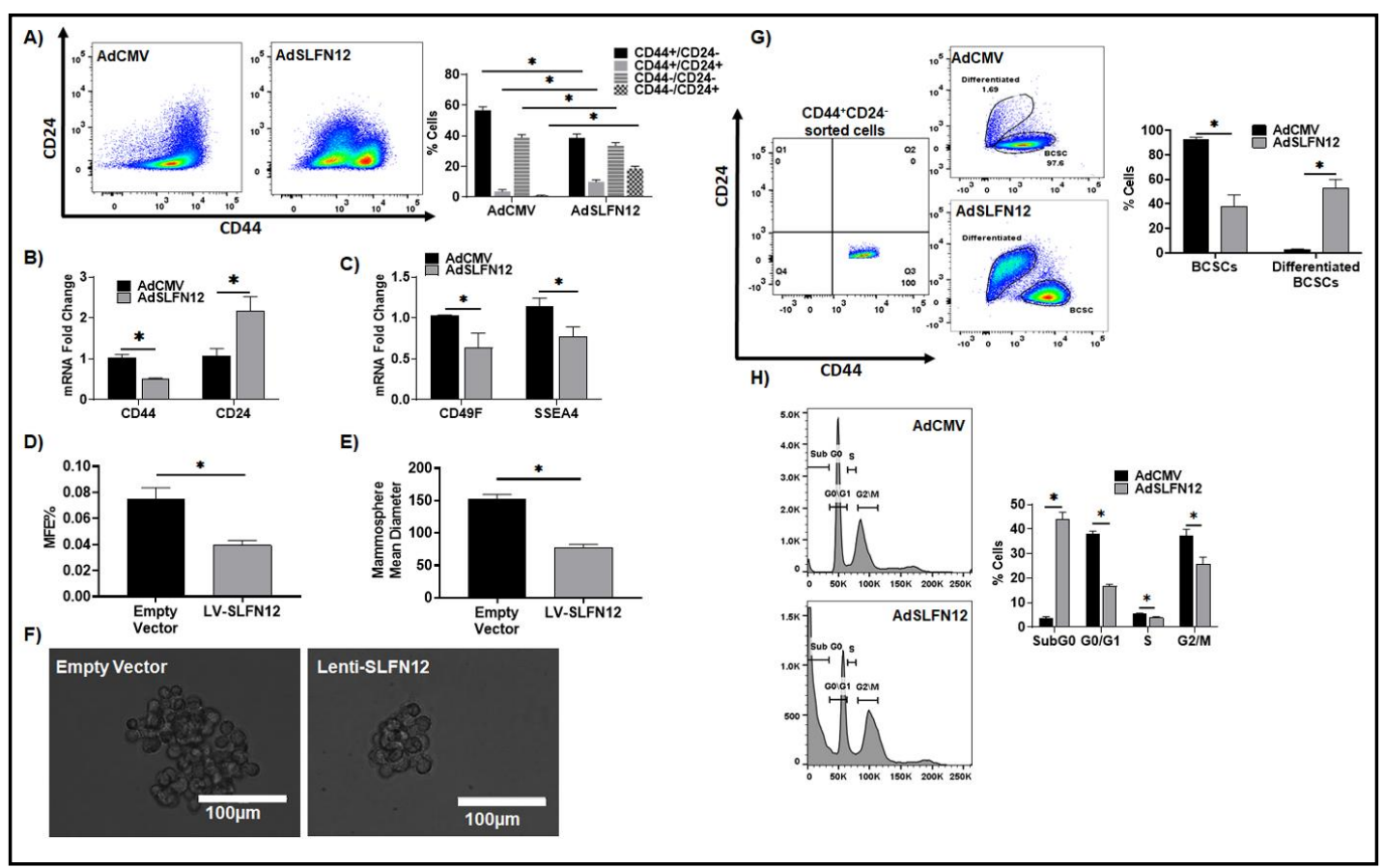

Fig. 4. Schlafen12 reduces MDA-MB-231 Breast Cancer Stem cell stemness. A) Flow cytometry analysis of MDA-MB-231 cells 96-hours after treatment with AdSLFN12 or AdCMV showing a reduction in CD44 ${ }^{+}$CD24 BCSCs population and an increase in differentiated non-BCSCs population (CD44+CD24+, CD44-CD24+, CD44CD24), ( $\left.n=6,{ }^{*} \mathrm{p}<0.05\right)$. B) SYBR Green qPCR analysis of mRNA levels of CD44 and CD24 and C) CD49F and SSEA4 in MDA-MB-231 cells 72 hours after treatment with either AdSLFN12 or AdCMV (RPLP0 used as a reference gene), data normalized to AdCMV group (CD44/24 n=6, CD49F/SSEA4 n=9, *p<0.05). D) Mammosphere formation efficiency (MFE \%) of SLFN12-expressing MDA-MB-231 stable clones, (MFE\% obtained by dividing the number of mammospheres over number of seeded cells then multiplied by 100), $\left(\mathrm{n}=18,{ }^{*} \mathrm{p}<0.05\right)$. E) Mammosphere formation assay of SLFN12-expressing MDA-MB-231 stable clones represent the mean \pm standard error diameter of the developed mammospheres. Mammospheres $<40 \mu \mathrm{m}$ were censored from the data. $\left(n=71,{ }^{*}<<0.05\right)$. F) Representative images of mammospheres developed from SLFN12-expressing MDA-MB-231 stable clones or control cells after 7 days incubation in MammoCult Medium. G) Flow cytometry analysis of sorted pure CD44+CD24- cell population from MDA-MB-231 cells treated with either AdSLFN12 or AdCMV for 96 hours $\left(n=8,{ }^{*} p<0.05\right)$. H) Cell cycle analysis by flow cytometry and Propidium iodide (PI) labeling of the sorted CD44 ${ }^{+} \mathrm{CD} 24^{-}$cells in (G) treated with either AdSLFN12 or AdCMV for 96 hours before analysis $\left(n=8,{ }^{*} \mathrm{p}<0.05\right)$. All error bars shown represent standard error of mean.

SLFN12 reduced ZEB1 protein by $49 \%$ and Slug by $41 \%$ while increasing Snail by $67 \%$ (Fig. $5 B, C)$. Since ZEB1 is a master regulator of EMT and TNBC aggressiveness [33] we focused on ZEB1 to further study how SLFN12 might modulate transcription factor protein disparately from mRNA. While AdSLFN12 decreased ZEB1 protein, knockdown of SLFN12 in MDAMB-231 cells (Supplementary Fig. 2) increased ZEB1 protein by 32\% (Fig. 5D, E, F).

To confirm that the SLFN12 effect on ZEB1 was not idiosyncratic to a single cell line, we exogenously expressed SLFN12 in BT549 and Hs578T cells. SLFN12 overexpression (Fig. 5G) reduced BT549 and Hs578T ZEB1 protein (Fig. 5H, I) similarly to MDA-MB-231 effects.

Schlafen12 inhibits the ZEB1 axis

To explore whether SLFN12 reduction in ZEB1 protein contributes to SLFN12 effects on differentiation, we co-expressed ZEB1 and SLFN12 in MDA-MB-231 cells. Co-expressing ZEB1 with SLFN12 (Fig. 6A) attenuated the effect of SLFN12 on E-cadherin mRNA to 79\% of control normalized to AdCMV vs. AdSLFN12 alone (231\% of control, normalized to AdCMV) (Fig. 6B), suggesting that SLFN12 induces E-cadherin expression at least in part by reducing 


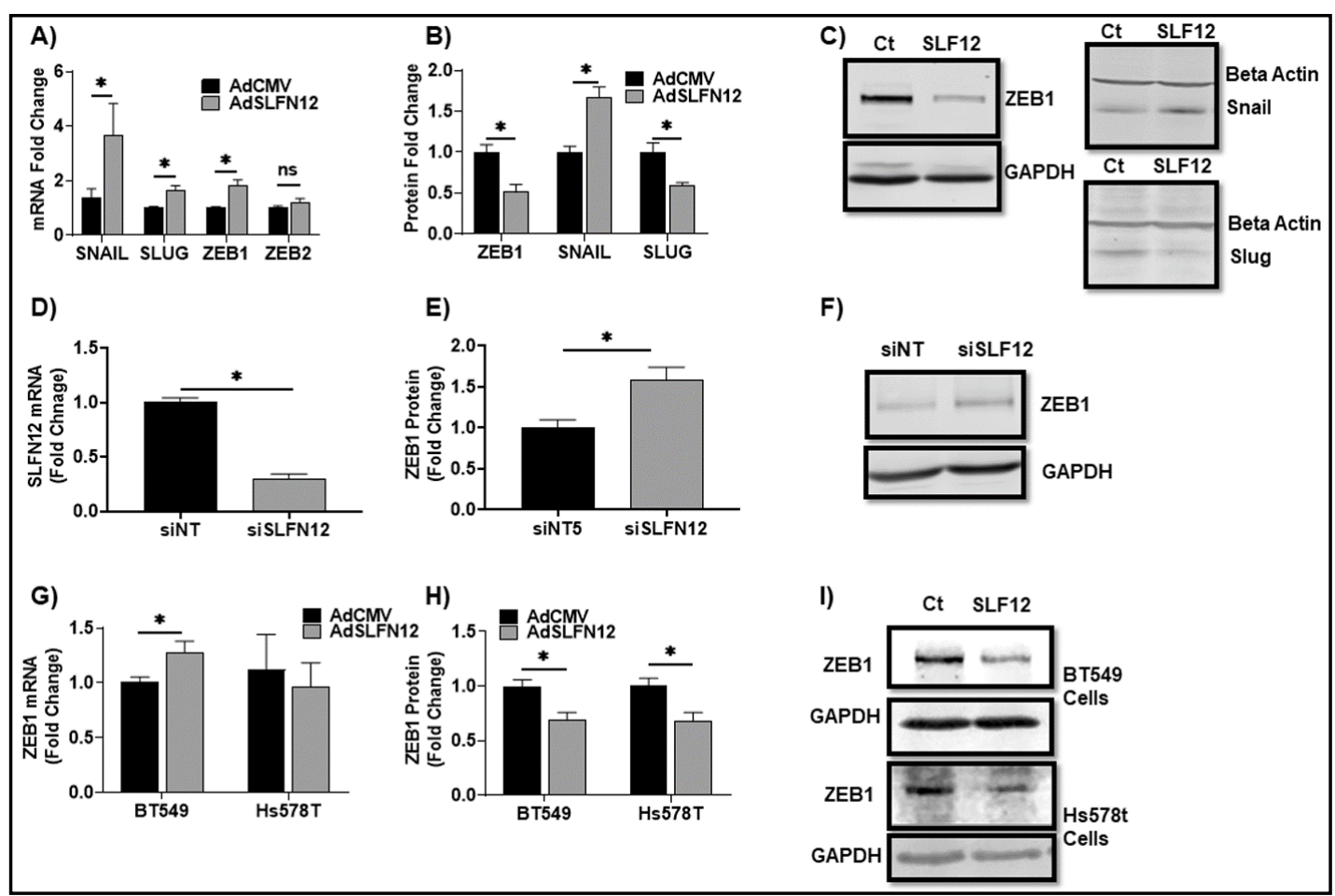

Fig. 5. Schlafen12 modulates ZEB1 levels. A) SYBR Green qPCR analysis of mRNA levels of Snail (n=9), Slug $(n=9)$, ZEB1 ( $n=15)$, and ZEB2 ( $=9$ ), (RPLP0 used as a reference gene) in MDA-MB-231 cells, 72 hours after treatment with either AdSLFN12 or AdCMV, data normalized to AdCMV group (*p<0.05). B) Protein levels of ZEB1( $n=17)$, Snail $(n=12)$, and Slug $(n=12)$ analyzed by western blot of MDA-MB-231 cells with C) Representative western blot images, 72 hours after treatment with AdSLFN12 or AdCMV (*p<0.05). (GAPDH and $\beta$-actin used as reference proteins). D) SYBR Green qPCR analysis of SLFN12 mRNA levels (RPLP0 used as reference gene), $\left(n=3,{ }^{*} p<0.05\right)$ and E) ZEB1 protein levels by western blot (GAPDH used as reference protein) in MDA-MB-231 cells 72 hours after treatment with either siSchlafen12 (siSLFN12) or nontargeting sequence ( $\operatorname{siNT})$, data normalized to siNT group, $\left(n=12,{ }^{*} \mathrm{p}<0.05\right)$ with F) Representative western blot images. G) SYBR Green qPCR analysis of ZEB1 mRNA levels and H) ZEB1 protein levels were analyzed by western blot in BT549 and Hs578T cells 72 hours after treatment with either AdSLFN12 or AdCMV, data normalized to AdCMV group (RPLP0 used as a reference gene and GAPDH as reference protein), ( $n=3-9$, ${ }^{*} \mathrm{p}<0.05$ ). I) Representative western blot images. All error bars shown represent standard error of mean.

ZEB1 protein. Co-expressing ZEB1 and SLFN12 also attenuated the antiproliferative effect of SLFN12 in MDA-MB-231, BT549, and Hs578T cells (Fig. 6C), suggesting that SLFN12 inhibits TNBC proliferation at least in part through modulating ZEB1 protein.

\section{SLFN12 reduces $Z E B 1$ protein translation}

Since SLFN12 exogenous expression reduced ZEB1 protein but increased ZEB1 mRNA, we hypothesized that SLFN12 regulates ZEB1 post-transcriptionally. We first measured the effect of SLFN12 on ZEB1 protein decay. We blocked MDA-MB-231 cells protein synthesis with cycloheximide, and measured ZEB1 protein over 0-24 hours. AdSLFN12 reduced the ZEB1 protein half-life vs. infection with AdCMV (17.93 $\pm 3.92 \mathrm{hr}$. vs. $40.25 \pm 10.91 \mathrm{hr}$.) (Fig. 6D, E). This suggests that SLFN12 regulates ZEB1 protein stability at least in part by modulating the rate of ZEB1 protein degradation.

To further evaluate whether SLFN12 influences ZEB1 proteasomal degradation, we blocked the proteasome with MG132. Treatment with $10 \mu \mathrm{m}$ of MG132 for twelve hours reduced ZEB1 protein in the presence of AdSLFN12 to 6\% of AdCMV controls vs. cells exposed to DMSO alone ( $71 \%$ of AdCMV controls, Fig. 6F). However, this increase was not comparable to the MG132 effect on ZEB1 in AdCMV controls which increased by 151\% vs DMSO-AdCMV 


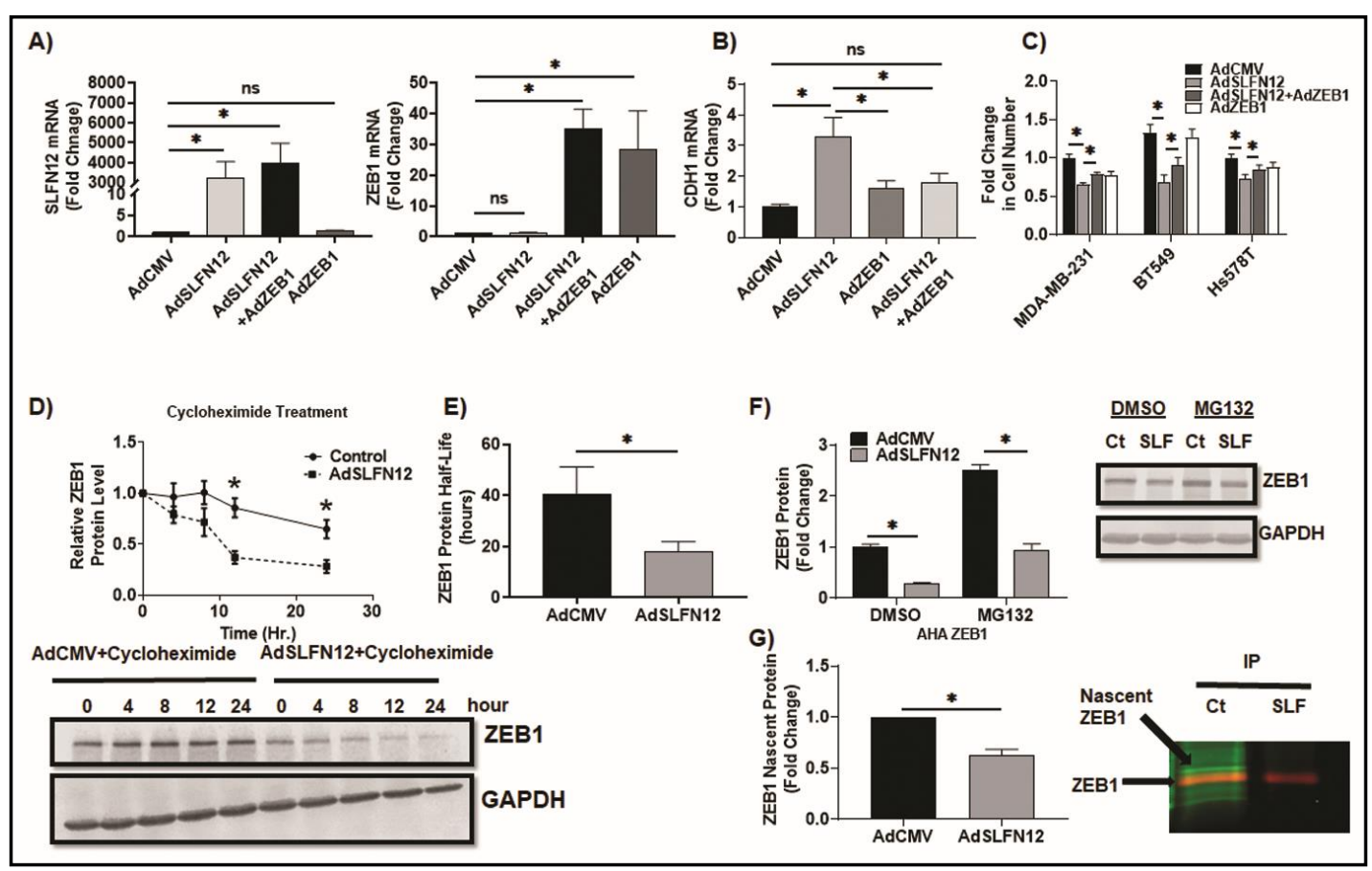

Fig. 6. Schlafen 12 acts at least in part through post-transcriptional modulation of ZEB1 protein. A) SYBR Green qPCR analysis of ZEB1, SLFN12 and B) E-cadherin (CDH1) mRNA levels in MDA-MB-231 cells, 72 hours after treatment with one of the following: AdSLFN12, AdZEB1, AdCMV or AdSLFN12 + AdZEB1. (Oneway ANOVA, $\left.n=16,{ }^{*} \mathrm{p}<0.05\right)$. C) Proliferation study using CCK8 assay in MDA-MB-231, BT549, and Hs578T cells, 72 hours after treatment with AdCMV, AdSLFN12, AdZEB1, AdSLFN12 + AdZEB1, data represent fold change in cell number normalized to the mean of AdCMV (MDA-MB-231 n=7, BT549 n=18, Hs578T n=11, ${ }^{*} \mathrm{p}<0.05$, student $\mathrm{t}$-test was used with Bonferroni correction). D) ZEB1 protein decay analysis by western blot in MDA-MB-231 cells at $0,4,8,12$, and 24 hours after treatment with $10 \mu \mathrm{g} / \mathrm{ml}$ cycloheximide in cells treated with AdSLFN12 or AdCMV for 72 hours. E) ZEB1 protein half-life analyzed by western blot in MDAMB-231 cells at after treatment with $10 \mu \mathrm{g} / \mathrm{ml}$ cycloheximide in cells treated with AdSLFN12 or AdCMV (as control) for 72 hours. ZEB1 half-life was calculated by online calculator (https://www.calculator.net/ half-life-calculator.html). F) ZEB1 protein levels analysis by western blot with representative blot images in MDA-MB-231 cells 72 hours after treatment with either AdCMV or AdSLFN12 in presence of DMSO or MG132 $(10 \mu \mathrm{M})$ for $12 \mathrm{hrs}$., GAPDH used as a reference protein and all data normalized to AdCMV+DMSO group ( $\left.\mathrm{n}=3,{ }^{*} \mathrm{p}<0.05\right)$. G) Translational assay of ZEB1 analyzed by western blot of MDA-MB-231 72 hours after treatment with either AdCMV or AdSLFN12 in presence of $50 \mu \mathrm{M}$ of L-AHA for 8 hours followed by ZEB1 immunoprecipitation, with representative blot image showing immunoprecipitated ZEB1 protein (red) and the nascent ZEB1 protein (green). Nascent ZEB1 band signal was normalized to immunoprecipitated ZEB1 band signal $\left(n=3,{ }^{*}<0.05\right)$. All error bars shown represent standard error of mean.

controls. Other proteasomal inhibitors (500nM bortezomib or $10 \mu \mathrm{M}$ carfilzomib) yielded comparable results (Supplementary Fig. 4). Thus, proteasomal inhibition attenuated the SLFN12 effect on ZEB1 protein but did not completely block SLFN12 reduction of ZEB1 protein, suggesting that SLFN12 regulates ZEB1 by another post-transcriptional mechanism in addition to proteasomal degradation.

Chloroquine failed to attenuate SLFN12 reduction of ZEB1 protein, indicating that SLFN12 does not regulate ZEB1 through autophagy-lysosomal degradation pathway (Supplementary Fig. 4). Therefore, we asked whether SLFN12 alters the translation of ZEB1. Metabolic labeling demonstrated that SLFN12 overexpression reduced ZEB1 protein translation by 38\% (Fig. 6G). Taken together, these findings suggest that SLFN12 regulates ZEB1 post-transcriptionally at both the levels of translation and proteasomal degradation, reducing ZEB1 protein but not ZEB1 mRNA. 


\section{Discussion}

The molecular pathways driving the aggressive biology of TNBC are not fully understood; therefore, no targeted therapy is available. TNBC are enriched with $\mathrm{CD} 44^{+} \mathrm{CD} 24$ - breast cancer stem cells that drive TNBC aggressiveness and chemoresistance [4, 5, 34]. SLFN12 expression appears to be a prognostic marker for TNBC. Moreover, although extrapolation from cell lines to in vivo biology can be challenging, our results suggest that SLFN12 reduces TNBC proliferation and invasiveness while increasing differentiation of TNBC cell populations. This appears to occur at least in part by the ability of SLFN12 to induce the differentiation of breast cancer stem cells (BCSCs) and by modulating protein levels of transcription factors such as ZEB1 via effects on both translation and proteasomal degradation.

Exogenous SLFN12 slowed proliferation and reduced invasion in TNBC cell lines. Although other human Schlafens (SLFN5, SLFN11, and SLFN13) reduce cancer cell proliferation [35-37], these are all long family Schlafens that localize to the nucleus and possess a helicase-like domain with the DNA-binding ability. In contrast, SLFN12 is an intermediate family Schlafen that lacks a nuclear targeting sequence or the helicase-like domain and localizes to the cytoplasm [38] with no reports of direct binding to DNA. This makes the anti-proliferative effect of SLFN12 mechanistically distinct from other human SLFN proteins. We previously reported that SLFN12 slows LNCaP and PC-3 prostate cancer proliferation [13] by an uncertain mechanism, but this is not true of all cells since exogenous SLFN12 did not reduce proliferation of MCF-7 (which are ER/PR+ breast cancer cells), suggesting a specific anti-proliferative effect of SLFN12 in TNBC. These results are consistent with our survival analysis that SLFN12 expression correlates with survival in TNBC but not in patients with $\mathrm{PR}+/ \mathrm{ER}+$ breast cancer.

Our results raise the possibility that SLFN12 may act in TNBC both by direct effects and by a reduction in the percentage of breast cancer stem cells within the cancer cell population. SLFN12 also appears to induce MDA-MB-231 cancer cell line differentiation, as indicated by increased E-cadherin and decreased vimentin expression. Vimentin has been reported to promote proliferation, invasion and mesenchymal status in MDA-MB-231 cells [39], and is upregulated and associated with poor prognosis in TNBC [8]. Downregulating vimentin reduces the proliferation, invasion, and mesenchymal characteristics of MDA-MB-231 cells [40]. Thus, the reduction of vimentin caused by exogenous SLFN12 could have contributed to the reduced proliferation, invasion and induced differentiation in MDA-MB-231 cells after SLFN12 overexpression. Exogenous SLFN12 also increased E-cadherin expression. E-cadherin inactivation is associated with poor prognosis in TNBC [41]. E-cadherin expression in MDA-BM-231 cells is both epigenetically reduced by hypermethylation and transcriptionally silenced [31], such loss of E-cadherin increases the invasiveness of these cells as the exogenous expression of E-cadherin in MDA-MB-231 cells reduces their metastatic potential and invasiveness while reestablishing epithelial polarity [42]. This would all be consistent with a model in which the increase in increased E-cadherin levels in response to SLFN12 also reduces the aggressiveness of MDA-MB-231. These results are consistent with a previous observation that SLFN12 increases E-cadherin protein levels in LNCaP prostate cancer cells [13], but extends these results to demonstrate an effect of SLFN12 on E-cadherin promoter activity and mRNA levels, which indicates a strong positive effect of SLFN12 on E-cadherin expression even when the gene is presumptively silenced.

Exogenous SLFN12 reduced the $\mathrm{CD} 44^{+} \mathrm{CD} 24^{-}$subpopulation within the overall population of MDA-MB-231 cells. SLFN12 overexpression in a specifically sorted CD $44^{+}$CD24subpopulation demonstrated that this was indeed a direct effect on breast cancer stem cells that induced BCSC to shift into a differentiated non-BCSC population $[28,43]$. The effect of SLFN12 on breast cancer stem cells was further reinforced by the observation that SLFN12 reduced mammosphere formation and sizes, established functional assays of stem cell capability [23]. The AdSLFN12 differentiating effect on BCSCs was accompanied by an alteration in cell cycle of the sorted BCSCs. AdSLFN12 caused more of the sorted CD44+CD24cells to shift into subG0/G1 phase, suggesting that Schlafen12 renders BCSCs cells more 
prone to apoptotic processes [44] while less cells progressed to the G2/M phase consistent with our observations of decreased cell proliferation and smaller size mammospheres in response to Schlafen12 overexpression.

Our results extend a previous observation that the murine slfn3 may reduce $\mathrm{CD} 44^{+} \mathrm{CD} 24$ cancer stem cells in FOLFOX-resistant colon cancer cells [45] in that we have studied the human SLFN12 here rather than expressing the 40\% homologous rodent slfn3 in human cells. Moreover, our results suggest a mechanism for the SLFN12 activity, which appears to occur at least in part by post-transcriptional downregulation of key transcription factors such as ZEB1 and Slug, each of which are known to be highly expressed in cancer stem cells $[46,47]$ and have been shown to regulate both stem cell biology and EMT [6, 8, 32, 48]. Furthermore, our flow sorting experiment demonstrates that SLFN12 acts directly upon TNBC cancer stem cells rather than exerting its effect by killing non-stem cells, inducing a secondary differentiation of the stem cells to replenish the non-stem cell compartment. A different study [16] has suggested that SLFN12 might induce intestinal epithelial differentiation by regulating deubiquitylation of the transcription factor cdx2 [16], but in our experiments cdx2 protein levels in MDA-MB-231 cells were not changed with exogenous SLFN12 (not shown). In contrast, we found that SLFN12 overexpression modulated ZEB1 protein levels in a post-transcriptional fashion in TNBC cell lines.

SLFN12 reduced both ZEB1 and Slug proteins through posttranscriptional regulation. The opposite effect on Snail protein would be consistent with a compensatory effect due to the loss of both ZEB1 and Slug, as Snail is an upstream regulator of ZEB1 [49]. ZEB1 knockdown has been reported to reduce cell proliferation, and tumor size in nude mice [50]. Posttranslational regulation of ZEB1 has been shown to modulate the cancer cell aggressiveness [7, 51]. Thus, these studies delineate a mechanism whereby the cytosolic intermediate SLFN12, lacking both a nuclear targeting sequence and the DNA-binding domain of the long SLFNs, can nevertheless strongly modulate differentiation by a different mechanism than that of the long SLFNs.

\section{Conclusion}

SLFN12 expression is prognostic for TNBC patients. SLFN12 reduces TNBC cell aggressiveness by reducing proliferation, and invasion, and by differentiating breast cancer stem cells. This effect is mediated at least in part by post-transcriptional regulation of ZEB1, a critical regulator of EMT and breast cancer stem cell differentiation. Schlafen12 may therefore be an important future target for TNBC therapy.

\section{Acknowledgements}

This work was supported in part by NIH 1 R01 DK096137 (MDB). Work in the flow cytometry core was supported in part by an Institutional Development Award (IDeA) from the National Institute of General Medical Sciences of the National Institutes of Health under grant numbers P20GM103442 and P20GM113123. The authors also thank Dr. Mary Ann Sens for her technical assistance.

\section{Statement of Ethics}

The authors have no ethical conflicts to disclose.

\section{Funding Sources}

This work was supported in part by the National Institute of Health (R01 DK096137 (MDB), 2014). Flow cytometry core was supported in part by an Institutional Development Award (IDeA) from the National Institute of General Medical Sciences of the National Institutes of Health under grant numbers P20GM103442 and P20GM113123. 


\section{Cellular Physiology and Biochemistry}

Cell Physiol Biochem 2019;53:999-1014

\begin{tabular}{l|l}
\hline DOI: 10.33594/000000191 & (c) 2019 The Author(s). Published by \\
\hline
\end{tabular}

Al-Marsoummi et al.: Schlafen12 Regulates Breast Cancer Stemness

Author Contributions

Dr. Sarmad Al-Marsoummi conceived, carried out experiments and analyzed data.

Dr. Emilie Vomhof-DeKrey carried out experiments and analyzed data.

Dr. Marc D. Basson conceived experiments and analyzed data.

All authors were involved in writing the manuscript and approved the final version.

\section{Disclosure Statement}

The authors have no conflicts of interest to declare.

\section{References}

1 Bianchini G, Balko JM, Mayer IA, Sanders ME, Gianni L: Triple-negative breast cancer: challenges and opportunities of a heterogeneous disease. Nat Rev Clin Oncol 2016;13:674-690.

2 Hudis CA, Gianni L: Triple-Negative Breast Cancer: An Unmet Medical Need. Oncologist 2011;16:1-11.

3 Abramson VG, Lehmann BD, Ballinger TJ, Pietenpol JA: Subtyping of triple-negative breast cancer: Implications for therapy. Cancer 2015;121:8-16.

4 Wang H, Wang L, Song Y, Wang S, Huang X, Xuan Q, Kang X, Zhang Q: CD44+/CD24- phenotype predicts a poor prognosis in triple-negative breast cancer. Oncol Lett 2017;14:5890-5898.

5 Ma F, Li H, Wang H, Shi X, Fan Y, Ding X, Lin C, Zhan Q, Qian H, Xu B: Enriched CD44+/CD24- population drives the aggressive phenotypes presented in triple-negative breast cancer (TNBC). Cancer Lett 2014;353:153-159.

6 Jang MH, Kim HJ, Kim EJ, Chung YR, Park SY: Expression of epithelial-mesenchymal transition-related markers in triple-negative breast cancer: ZEB1 as a potential biomarker for poor clinical outcome. Hum Pathol 2015;46:1267-1274.

7 Maturi V, Enroth S, Heldin CH, Moustakas A: Genome-wide binding of transcription factor ZEB1 in triplenegative breast cancer cells. J Cell Physiol 2018;233:7113-7127.

8 Karihtala P, Auvinen P, Kauppila S, Haapasaari KM, Jukkola Vuorinen A, Soini Y: Vimentin, zeb1 and Sip1 are up-regulated in triple-negative and basal-like breast cancers: Association with an aggressive tumour phenotype. Breast Cancer Res Treat 2013;138:81-90.

9 Geradts J, De Herreros AG, Su Z, Burchette J, Broadwater G, Bachelder RE: Nuclear Snail1 and nuclear ZEB1 protein expression in invasive and intraductal human breast carcinomas. Hum Pathol 2011;42:1125-1131.

10 Liu F, Zhou P, Wang Q Zhang M, Li D: The Schlafen family: complex roles in different cell types and virus replication. Cell Biol Int 2017;42:2-8.

11 Uhlen M, Zhang C, Lee S, Sjöstedt E, Fagerberg L, Bidkhori G, Benfeitas R, Arif M, Liu Z, Edfors F, Sanli K, Feilitzen K, Oksvold P, Lundberg E, Hober S, Nilsson P, Mattsson J, Schwenk J, Brunnström H, et al.: A pathology atlas of the human cancer transcriptome. Science 2017;357:eaan2507.

12 Sassano A, Mavrommatis E, Arslan AD, Kroczynska B, Beauchamp EM, Khuon S, Chew TL, Green KJ, Munshi HG, Verma AK, Plataniasa LC: Human Schlafen 5 (SLFN5) Is a Regulator of Motility and Invasiveness of Renal Cell Carcinoma Cells. Mol Cell Biol 2015;35:2684-2698.

13 Kovalenko PL, Basson MD: Schlafen 12 expression modulates prostate cancer cell differentiation. J Surg Res 2014;190:177-184.

14 Bustos O, Naik S, Ayers G, Casola C, Perez-Lamigueiro MA, Chippindale PT, Prithama E, Casa Esperóna E: Evolution of the Schlafen genes, a gene family associated with embryonic lethality, meiotic drive, immune processes and orthopoxvirus virulence. Gene 2009;447:1-11.

15 Chaturvedi L, Sun K, Walsh MF, Kuhn LA, Basson MD: The P-loop region of Schlafen 3 acts within the cytosol to induce differentiation of human Caco-2 intestinal epithelial cells. Biochim Biophys Acta 2014;1843:3029-3037.

16 Basson MD, Wang Q, Chaturvedi LS, More S, Vomhof-DeKrey EE, AlMarsoummi S, Sun K, Kuhn L, Kovalenko P, Kiupel M: Schlafen 12 Interaction with SerpinB12 and Deubiquitylases Drives Human Enterocyte Differentiation. Cell Physiol Biochem 2018;48:1274-1290. 
17 Kovalenko PL, Yuan L, Sun K, Kunovska L, Seregin S, Amalfitano A, Basson MD: Regulation of epithelial differentiation in rat intestine by intraluminal delivery of an adenoviral vector or silencing RNA coding for Schlafen 3. PLoS One 2013;8:e79745.

18 Walsh MF, Hermann R, Lee JH, Chaturvedi L, Basson MD: Schlafen 3 Mediates the Differentiating Effects of Cdx2 in Rat IEC-Cdx2L1 Enterocytes. J Invest Surg 2015;28:202-207.

19 Schwarz DA, Katayama CD, Hedrick SM: Schlafen, a New Family of Growth Regulatory Genes that Affect Thymocyte Development. Immunity 1998;9:657-668.

20 de Waal L, Lewis TA, Rees MG, Tsherniak A, Wu X, Choi PS, Gechijian L, Hartigan C, Faloon PW, Hickey MJ, Tolliday N, Carr SA, Clemons PA, Munoz B, Wagner BK, Shamji AF, Koehler AN, Schenone M, Burgin AB, Schreiber SL, et al.: Identification of cancer-cytotoxic modulators of PDE3A by predictive chemogenomics. Nat Chem Biol 2016;12:102-108.

21 Tissue expression of SLFN12 - Summary - The Human Protein Atlas [Internet] [cited 2017 Nov 28]. URL: https://www.proteinatlas.org/ENSG00000172123-SLFN12/tissue.

22 Kaplan Meier-plotter [Breast cancer] [Internet] [cited 2017 Oct 22]. URL: http://kmplot.com/analysis/ index.php?p=service \&start $=1$.

23 Shaw FL, Harrison H, Spence K, Ablett MP, Simões BM, Farnie G, Clarke RB: A detailed mammosphere assay protocol for the quantification of breast stem cell activity. J Mammary Gland Biol Neoplasia 2012;17:111117.

24 Györffy B, Lanczky A, Eklund AC, Denkert C, Budczies J, Li Q Szallasi Z: An online survival analysis tool to rapidly assess the effect of 22,277 genes on breast cancer prognosis using microarray data of 1,809 patients. Breast Cancer Res Treat 2010;123:725-731.

25 Jézéquel P, Campone M, Gouraud W, Guérin-Charbonnel C, Leux C, Ricolleau G, Campion L: Bc-GenExMiner: An easy-to-use online platform for gene prognostic analyses in breast cancer. Breast Cancer Res Treat 2012;131:765-775.

26 EMBL-EBI. Expression Atlas [Internet]. Int Cancer Genome Proj Pan-Cancer Anal Whole Genomes 2019 [cited 2019 May 13]. URL: https://www.ebi.ac.uk/gxa/experiments/E-MTAB-5200/Results?geneQuery=\% 5B\%7B\%22value\%22\%3A\%22ENSG00000172123\%22\%7D\%5D\&filterFactors=\%7B\%22ORGANISM_PAR T\%22\%3A\%5B\%22breast\%22\%5D\%7D.

27 Ivaska J, Peuhu E, Mai A, Wärri A, Virtakoivu R: Epithelial vimentin plays a functional role in mammary gland development. Development 2017;144:4103-4113.

28 Pham PV, Phan NL, Nguyen NT, Truong NH, Duong TT, Le DV, Truong KD, Phan NK: Differentiation of breast cancer stem cells by knockdown of CD44: promising differentiation therapy. J Transl Med 2011;9:209.

29 Gómez-Miragaya J, Palafox M, Paré L, Yoldi G, Ferrer I, Vila S, Galván P, Pellegrini P, Pérez Montoyo H, Igea A, Muñoz P, Esteller M, Nebreda A, Urruticoechea A, Morilla I, Pernas S, Climent F, Soler Monso M, Petit A, et al.: Resistance to Taxanes in Triple-Negative Breast Cancer Associates with the Dynamics of a CD49f+ Tumor-Initiating Population. Stem Cell Reports 2017;8:1392-407.

30 Aloia A, Petrova E, Tomiuk S, Bissels U, Déas O, Saini M, Zickgraf F, Wagner S, Spaich S, Sütterlin M, Schneeweiss A, Reitberger M, Rüberg S, Gerstmayer B, Agorku D, Knöbel S, Terranegra A, Falleni M, Soldati L, Sprick M, et al.: The sialyl-glycolipid stage-specific embryonic antigen 4 marks a subpopulation of chemotherapy-resistant breast cancer cells with mesenchymal features. Breast Cancer Res 2015;17:146.

31 Lombaerts M, van Wezel T, Philippo K, Dierssen JW, Zimmerman RM, Oosting J, van Eijk R, Eilers PH, van de Water B, Cornelisse CJ, Cleton Jansen AM: E-cadherin transcriptional downregulation by promoter methylation but not mutation is related to epithelial to mesenchymal transition in breast cancer cell lines. Br J Cancer 2006;94:661-671.

32 Cho HJ, Oh N, Park JH, Kim KS, Kim HK, Lee E, Hwang S, Kim SJ, Park KS: ZEB1 Collaborates with ELK3 to Repress E-Cadherin Expression in Triple-Negative Breast Cancer Cells. Mol Cancer Res 2019;17:22572266.

33 Graham TR, Yacoub R, Taliaferro-Smith L, Osunkoya AO, Odero-Marah VA, Liu T, Sean Kimbro K, Sharma D, O’Regan R: Reciprocal regulation of ZEB1 and AR in triple negative breast cancer cells. Breast Cancer Res Treat 2010;123:139-147.

34 Liu M, Liu Y, Deng L, Wang D, He X, Zhou L, Wicha MS, Bai F, Liu S: Transcriptional profiles of different states of cancer stem cells in triple-negative breast cancer. Mol Cancer 2018;17:65.

35 Brady G, Boggan L, Bowie A, O'Neill LAJ. Schlafen-1 causes a cell cycle arrest by inhibiting induction of cyclin D1. J Biol Chem 2005;280:30723-30734. 


\section{Cellular Physiology and Biochemistry}

Cell Physiol Biochem 2019;53:999-1014

\begin{tabular}{l|l}
\hline DOI: 10.33594/000000191 & (c) 2019 The Author(s). Published by \\
\hline
\end{tabular}

Al-Marsoummi et al.: Schlafen12 Regulates Breast Cancer Stemness

36 Zoppoli G, Regairaz M, Leo E, Reinhold WC, Varma S, Ballestrero A, Doroshow JH, Pommier Y: Putative DNA/ RNA helicase Schlafen-11 (SLFN11) sensitizes cancer cells to DNA-damaging agents. Proc Natl Acad Sci 2012;109:15030-15035.

37 Wan G, Liu Y, Zhu J, Guo L, Li C, Yang Y, Gu X, Deng LL, Lu C: SLFN5 suppresses cancer cell migration and invasion by inhibiting MT1-MMP expression via AKT/GSK-3 $\beta / \beta$-catenin pathway. Cell Signal 2019;59:1-12.

38 Neumann B, Zhao L, Murphy K, Gonda TJ: Subcellular localization of the Schlafen protein family. Biochem Biophys Res Commun 2008;370:62-66.

39 Mendez MG, Kojima SI, Goldman RD: Vimentin induces changes in cell shape, motility, and adhesion during the epithelial to mesenchymal transition. FASEB J 2010;24:1838-1851.

40 Liu CY, Lin HH, Tang MJ, Wang YK: Vimentin contributes to epithelial-mesenchymal transition cancer cell mechanics by mediating cytoskeletal organization and focal adhesion maturation. Oncotarget 2015;6:15966-15983.

41 Shen T, Zhang K, Siegal GP, Wei S: Prognostic value of E-cadherin and $\beta$-catenin in triple-negative breast cancer. Am J Clin Pathol 2016;146:603-610.

42 Mbalaviele G, Dunstan CR, Sasaki A, Williams PJ, Mundy GR, Yoneda T: E-cadherin expression in human breast cancer cells suppresses the development of osteolytic bone metastases in an experimental metastasis model. Cancer Res 1996;56:4063-4070.

43 Al-Hajj M, Wicha MS, Benito-Hernandez A, Morrison SJ, Clarke MF: Prospective identification of tumorigenic breast cancer cells. Proc Natl Acad Sci 2003;100:3983-3988.

44 Aithal MGS, Rajeswari N. Bacoside A: Induced Sub-G0 Arrest and Early Apoptosis in Human Glioblastoma Cell Line U-87 MG through Notch Signaling Pathway. Brain Tumor Res Treat 2019;7:25-32.

45 Oh PS, Patel VB, Sanders MA, Kanwar SS, Yu Y, Nautiyal J, Patel BB, Majumdar AP: Schlafen-3 decreases cancer stem cell marker expression and autocrine/juxtacrine signaling in FOLFOX-resistant colon cancer cells. Am J Physiol Gastrointest Liver Physiol 2011;301:G347-355.

46 Bhat Nakshatri P, Appaiah H, Ballas C, Pick Franke P, Goulet R, Badve S, Srour EF, Nakshatri H: SLUG/SNAI2 and Tumor Necrosis Factor Generate Breast Cells With CD44+/CD24-Phenotype. BMC Cancer 2010;6:411.

47 Zhou C, Jiang H, Zhang Z, Zhang G, Wang H, Zhang Q, Sun P, Xiang R, Yang S: ZEB1 confers stem cell-like properties in breast cancer by targeting neurogenin-3. Oncotarget 2017;8:54388-54401.

48 Su Y, Pogash TJ, Nguyen TD, Russo J: Development and characterization of two human triple-negative breast cancer cell lines with highly tumorigenic and metastatic capabilities. Cancer Med 2016;5:558-573.

49 Dave N, Guaita Esteruelas S, Gutarra S, Frias À, Beltran M, Peiró S, De Herreros AG: Functional cooperation between snail1 and twist in the regulation of ZEB1 expression during epithelial to mesenchymal transition. J Biol Chem 2011;286:12024-12032.

50 Arima Y, Hayashi H, Sasaki M, Hosonaga M, Goto TM, Chiyoda T, Kuninaka S, Shibata T, Ohata H, Nakagama $\mathrm{H}$, Taya Y, Saya H: Induction of ZEB proteins by inactivation of RB protein is key determinant of mesenchymal phenotype of breast cancer. J Biol Chem 2012;287:7896-7906.

51 Zhou Z, Zhang P, Hu X, Kim J, Yao F, Xiao Z, Zeng L, Chang L, Sun Y, Ma L: USP51 promotes deubiquitination and stabilization of ZEB1. Am J Cancer Res 2017;7:2020-2031. 